\title{
Klinische und experimentelle Forschung sind gleichwertig
}

$\mathrm{N}$ ur ein halbes Jahr nach dem großen Kongreß in Freiburg konnten wir eine erfreuliche Frühjahrstagung der Deutschen Gesellschaft für Allergologie und klinische Immunologie (DGAI) in Mainz besuchen und hochinteressante neue Forschungsergebnisse aus verschiedenen Arbeitsgruppen in Deutschland, den Niederlanden und der Schweiz hören. Von Prof. SchultzeWerninghaus und Prof. Knop in bewährter Weise organisiert, gab es eine ausgewogene Mischung von experimenteller und klinischer Forschung. Das ist wichtig. Denn diese beiden Arten von wissenschaftlicher Arbeit sind gleichwertig.

Es ist nicht so, daß eine experimentelle Studie prinzipiell der klinischen Beobachtung überlegen sein muß, obgleich dies von manchen jungen Forschern immer wieder so vermutet wird. Allergologie ist eine klinische Disziplin und lebt von der Anwendung der experimentellen, vorwiegend der immunologischen Forschung am Patienten. Die Überprüfung aller aufregenden und schönen Hypothesen aus tierexperimentellen und zellbiologischen Studien gelingt ja erst am Menschen. Die Stunde der Wahrheit schlägt mit der Frage nach der klinischen Relevanz.

Es gibt dementsprechend verschiedene Ebenen der experimentellen Forschung, genauso wie der klinischen Forschung. Die Überprüfung der Wirksamkeit eines neuen Therapieprinzips oder Pharmakons gelingt nur durch die saubere, nach allen Regeln der Kunst durchgeführte kontrollierte klinische Studie. Ausgangspunkt für neue Forschungsarbeiten sind aber auch Einzelbeobachtungen. Deshalb sind exzellent aufgearbeitete exemplarische Fälle einer wissenschaftlichen Kasuistik oft origineller und lehrreicher als wechselnde Zytokin- muster in Zellsuspensionen oder Mäusen. Die wissenschaftliche Arbeit einer guten Kasuistik ist aber auch genauso intensiv. Schlampige Fallvorstellungen untergraben das Image des Klinikers und verstärken Vorurteile bei Theoretikern, daß es sich bei Kasuistiken ja nur um „Plauderstunden am Kamin" handele.

Die exakte Diagnostik, Klassifikation und Dokumentation des klinischen Zustandsbildes stellt die unabdingbare Voraussetzung für experimentelle Forschung mit modernen aufwendigen Labormethoden dar. Leider besteht oft ein Ungleichgewicht in der wissenschaftlichen Potenz der klinischen und experimentellen Partner. Mit modernster Molekularbiologie und aufwendigsten Techniken werden Daten erarbeitet, ohne daß diese einem klargeschnittenen klinischen $\mathrm{Zu}$ standsbild einschließlich Schweregrad, Krankheitsphase etc. zuzuordnen wären. Auf der anderen Seite werden in klinischen Studien mit großem Aufwand Befunde erhoben, Blutabnahmen durchgeführt und wesentliche weiterführende Parameter vergessen.

Die aktiven Arbeitsgruppen in unserer Gesellschaft vertreten beides, dies ist auch in Mainz wieder ganz klar geworden. Ohne klinischen Bezug wird die experimentelle Allergologie in reine Zell- und Molekularbiologie abdriften, die eigentlich überall und unter jedem Vorzeichen durchgeführt werden kann.

In den Zeiten knapper werdender Ressourcen in Klinik und Forschung kommt es zu härteren Konkurrenz-

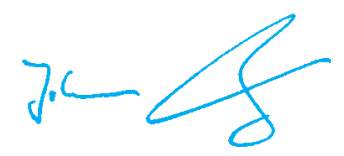

Prof. Dr. Dr. J. Ring kämpfen. Die Allergologengemeinde ist so klein und in ihrer Interdisziplinarität so heterogen, daß wir es uns nicht leisten können, einander schlecht zu machen. Darauf warten unsere „Feinde“, das sind nicht andere Wissenschaftler oder andere Fachgruppen, nein, der eigentliche und zukunftsentscheidende Kampf spielt sich mit den Forschungsgegnern, den Anti-Spezialisten, den ,Alternativen" ab, die unsere Arbeit insgesamt in Frage stellen oder verhindern wollen.

Vorstand, Beirat und Sektionsvorsitzende der DGAI haben deshalb auf der Sitzung in Mainz beschlossen, die in Freiburg bereits vorgestellten Qualitätsrichtlinien im Rahmen des Konzepts zur allergologischen Versorgung der Bevölkerung in Deutschland weiter $\mathrm{zu}$ verbessern und zu unterstützen, ein Curriculum der verbesserten Weiterbildung zum Allergologen auf der Basis der fachbezogenen Allergologie - wie sie sich in Mitteleuropa durchgesetzt hat auch in der Europäischen Union zu vertreten und schließlich Leitlinien der Diagnostik und Therapie für die wichtigsten Krankheitsbilder zu erarbeiten.

Erfreulich ist auch zu berichten, daß es wieder eine Preisverleihung gab: Der Preis der Stiftung Kanert für Allergieforschung ging an Frau Dr. Schierhorn, aus der Arbeitsgruppe von Prof. Kunkel, Berlin, für ein geplantes Forschungsprojekt zum Einfluß von Tachykinin-Antagonisten bei der neurogenen Entzündung der Nasenschleimhaut.

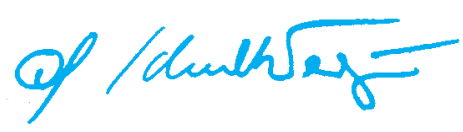

Prof. Dr. G. Schultze-Werninghaus 